\title{
Incidence, predictors and prognostic significance of thromboembolic disease in patients with advanced $A L K$ - rearranged non-small cell lung cancer
}

\author{
To the Editor:
}

Thromboembolic disease is fairly common in patients with lung cancer [1-3]. This incidence seems to be higher in patients with lung adenocarcinomas [4], with approximately $15 \%$ of those with advanced stage disease developing venous thromboembolisms (VTE) during the whole course of their disease [5-7]. Pulmonary adenocarcinomas are a heterogeneous group of diseases that can be stratified according to the presence of major oncogenic driver alterations. Anaplastic lymphoma kinase $(A L K)$ rearrangements are detected in approximately $4 \%$ of these cases [8]. Isolated reports have suggested that patients bearing $A L K$-rearranged tumours might have a higher than expected incidence of thromboembolisms $[9,10]$. In the present study, we have analysed the incidence, predictors and prognostic significance of thromboembolic events in a large, multi-institutional and homogeneous cohort of advanced stage patients with $A L K$-rearranged lung cancers from Spain and Portugal. Our primary objective was to estimate the incidence of thromboembolic events and their association with overall survival in these patients.

A centralised institutional ethics committee approval at the 12 de Octubre University Hospital valid for all Spanish centres, and an institutional ethics committee approval at the Portuguese Institute of Oncology of Porto, were obtained before the study was initiated. We retrospectively selected all consecutive patients diagnosed with advanced stage (stages III and IV) $A L K$ fusion positive non-small cell lung cancers (NSCLCs) between January 2012 and December 2016. Data were contributed by 29 Medical Centres from Spain and one from Portugal. ALK positivity was determined according to local standard protocols in each institution. We excluded patients with neuroendocrine tumours and patients on therapeutic doses of anticoagulants prior to advanced stage cancer diagnosis. We defined a thromboembolic event as any venous or arterial thromboembolism, documented by imaging studies, that occurred at the time or after advanced stage cancer diagnosis. In addition to thromboembolic events, collected during the whole patients' follow-up period, we collected baseline information (within 1 month of advanced stage cancer diagnosis) of several clinical and analytical variables of interest.

We included 241 ALK-rearranged NSCLCs in this study. The median age was 56 years (range 1784 years). Half of the patients were never smokers (52\%), and most had stage IV pulmonary adenocarcinomas $(n=204,85 \%)$. Baseline brain and liver metastasis were detected in $22 \%$ and $25 \%$ of the patients, respectively. 17 patients $(7 \%)$ and 185 patients $(77 \%)$ had high and intermediate Khorana risk scores (KRS) [11] respectively. The median follow-up of our study population was 19 months (range 059 months), and 127 (53\%) of the patients died. The median follow-up of alive patients was 30 months (range 4-49 months). The estimated median overall survival was 26 months for the entire series, and 31 months for those patients that received ALK tyrosine kinase inhibitors (TKIs) (n=207, 86\%).

73 patients (30\%) experienced thromboembolic complications. 71 out of 73 patients $(97 \%)$ had VTE, with pulmonary embolism as the most frequent location $(n=30 ; 41 \%)$. Four patients $(5 \%)$ developed arterial thromboembolisms, two of them with concurrent VTE. Thromboembolic events occurred within the first 6 months from cancer diagnosis in 54 patients (74\%), of whom $24(33 \%)$ were detected within the first

@ERSpublications

High incidence and prognostic relevance of thromboembolic disease in patients with ALK-rearranged NSCLCs http://ow.ly/DEZr30j6kC8

Cite this article as: Zugazagoitia J, Biosca $\mathrm{M}$, Oliveira $\mathrm{J}$, et al. Incidence, predictors and prognostic significance of thromboembolic disease in patients with advanced $A L K$-rearranged non-small cell lung cancer. Eur Respir J 2018; 51: 1702431 [https://doi.org/10.1183/13993003.02431-2017]. 
month from diagnosis. 22 patients (30\%) were deemed to have incidental (asymptomatic or clinically unsuspected) thromboembolisms. 12 patients (16\%) developed recurrent thrombosis (seven while receiving appropriate therapeutic anticoagulation). The mortality directly attributed to thromboembolic disease in this series was $2 \%(n=5)$.

To study the association between baseline characteristics and thromboembolic disease, we first conducted a univariate competing risk regression analysis [12] including gender, age, body mass index, performance status, smoking history, histology, tumour stage, central nervous system metastasis, liver metastasis, number of metastatic sites, haemoglobin, leukocyte, platelet and albumin counts, INR range and KRS. In this analysis, the presence of baseline liver metastases, leucocyte counts $>11000$ cells per $\mathrm{mm}^{3}$, three or more metastatic organ involvement and a high KRS (HR 2.82, CI 95\% 1.48-5.37; p=0.002) were significantly associated with increased risk of thromboembolic disease. In the multivariate model (in which we did not include KRS because it already incorporates leukocyte counts), only liver metastases and leukocytosis remained as independent predictors of thromboembolic disease, with corresponding HR of 1.85 (CI 95\% 1.09-3.15; p=0.021) and HR of 2.34 (CI 95\% 1.43-3.82; $\mathrm{p}=0.001$ ), respectively.

Patients that experienced thromboembolic events at any time point had shorter median overall survival (20 months) than patients without thrombosis (36 months) $(\mathrm{p}=0.035)$ (figure 1a). Patients with thromboembolic events at baseline $(n=24)$ had a median overall survival of 15 months (figure $1 b)$. Patients that experienced recurrent thromboembolisms $(n=12)$ had a median overall survival of 10 months, without statistically significant differences when compared with those without recurrent thromboembolism $(n=61$; 21 months) ( $p=0.369)$. Considering the development of thrombotic events as a time-dependent covariate, thromboembolic disease was significantly associated with an increased risk of death (HR 2.22, CI 95\% 1.53-3.21; $\mathrm{p}=0.000$ ). We conducted a multivariate Cox regression analysis including smoking history, comorbidities, leukocytosis, liver metastasis, performance status and ALK TKI treatment as fixed covariates, and thromboembolic disease as a time-varying covariate. In this analysis, thromboembolic disease remained as an independent predictor of overall survival with HR 1.70 (CI 95\% 1.10-2.62; $\mathrm{p}=0.016)$ (figure 1c). The presence of thromboembolic disease at baseline $(n=24)$ was associated with a numerically nonsignificant increased risk of death (HR 1.67, CI 95\% 0.96-2.91; $\mathrm{p}=0.068$ ).
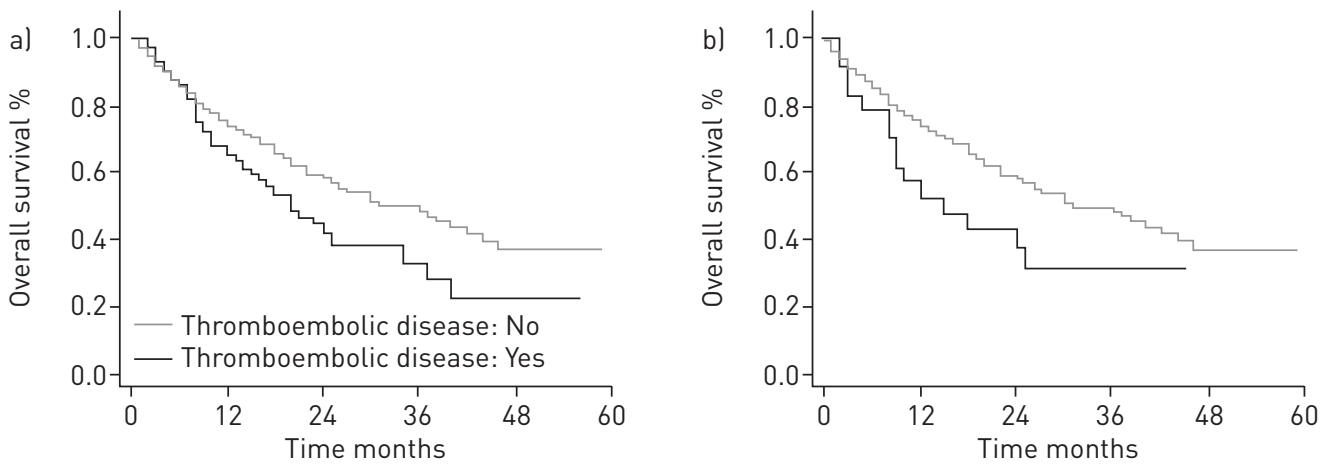

\begin{tabular}{lcccccc}
\multicolumn{2}{l}{ Number at risk } \\
No & 167 & 123 & 70 & 35 & 12 & 0 \\
Yes & 73 & 48 & 27 & 11 & 2 & 0
\end{tabular}

$\begin{array}{lll}\text { Number at risk } \\ \text { No } & 168 & 123 \\ \text { Yes } & 24 & 12\end{array}$

c)

\begin{tabular}{lcc}
\hline Variable & Multivariate HR (95\% CI) & p-value \\
\hline Thromboembolic disease & $1.70(1.10-2.62)$ & 0.016 \\
Tyrosine-kinase inhibitor treatment & $0.60(0.37-0.97)$ & 0.041 \\
Smoking history & $1.60(1.11-2.31)$ & 0.011 \\
>1 comorbidities & $2.20(1.31-3.71)$ & 0.003 \\
$>11000$ leukocytes per $\mathrm{mm}^{3}$ & $1.68(1.09-2.59)$ & 0.019 \\
Liver metastasis & $1.49(0.99-2.25)$ & 0.054 \\
Performance status & $1.76(1.36-2.27)$ & 0.000 \\
\hline
\end{tabular}

FIGURE 1 Prognostic significance of thromboembolic disease in advanced ALK-positive non-small cell lung cancer patients. a) Kaplan-Meier curve for overall survival for patients with and without thromboembolic disease. b) Kaplan-Meier curve for overall survival for patients with baseline thromboembolic disease. c) Multivariate analysis showing the association of thromboembolic disease and overall survival. 
In the present study, we show that thromboembolic disease is a frequent complication in patients with advanced-stage $A L K$-positive NSCLCs (30\%), particularly in the presence of baseline liver metastasis or leukocytosis $(\sim 50 \%)$, and it is associated with a lower overall survival in this particular subtype of lung cancer. This study was not designed to compare the incidence of thromboembolic events between different molecular subtypes of lung cancers. However, the high incidence observed here is consistent with what has been reported in smaller ALK-positive cohorts $[9,10]$, and exceeds the observed in other subtypes of lung cancers $[2,3,5-7]$. We found that thromboembolic disease was an independent prognostic factor in advanced $A L K$-positive NSCLCs. Although the mortality rate of thromboembolic complications might have been underestimated in this study [13], the differences in survival do not seem to be directly related with the fatality of thromboembolic events themselves $(2 \%$ of mortality attributed to thromboembolic disease in this cohort). In the present study, both leukocytosis and liver metastasis significantly predicted both the occurrence of thrombotic complications and reduced overall survival (figure 1c). A relatively low proportion of patients had progressive disease at the time of thromboembolic complications (22\%), but we cannot definitely exclude that the association of thromboembolic disease with lower overall survival was confounded by subsequent development of liver metastasis and/or leukocytosis in some patients, as we only had baseline information for these variables.

Our findings have relevant implications for clinical practice. First, physicians should actively examine for the presence of signs or symptoms of thrombosis at diagnosis and during follow-up of these patients, and accordingly rule out the existence of thromboembolic disease with appropriate imaging studies. Additional surveillance strategies in high risk patients, including those with leukocytosis or liver metastasis, would need to be eventually considered. Second, these results justify investigation, in the context of a randomised trial, of the clinical benefit, in terms of thromboembolisms risk reduction and potentially in overall survival improvement, of adding prophylactic doses of anticoagulants to standard anticancer treatments in these patients. And ultimately, with median overall survival estimates exceeding 4 to 5 years with the incorporation of novel-generation ALK TKIs into the clinic [14, 15], thromboembolic disease will be one of the main cancer-related complication during the whole course of the disease in these patients.

Jon Zugazagoitia ${ }^{1,2,3,35}$, Mercedes Biosca ${ }^{4}$, Julio Oliveira $^{5}$, María Eugenia Olmedo ${ }^{6}$, Manuel Dómine ${ }^{7}$, Ernest Nadal ${ }^{8,9}$, José Carlos Ruffinelli ${ }^{8}$, Nerea Muñoz ${ }^{1}$, Ana María Luna ${ }^{10}$, Berta Hernández ${ }^{11}$, Maite Martínez ${ }^{11}$, Iria Gallego ${ }^{12}$, Eva Martínez de Castro ${ }^{13}$, Carme Font $^{14}$, Virginia Calvo ${ }^{15}$, Virginia Martínez-Marín ${ }^{16}$, Jesús Corral ${ }^{17}$, Esther Noguerón ${ }^{18}$, Rebeca Mondéjar ${ }^{19}$, Ignacio García Escobar ${ }^{20}$, Carmen Salvador-Coloma ${ }^{21}$, Óscar Juan ${ }^{21}$, Manuel Sánchez Cánovas ${ }^{22}$, Javier Valdivia ${ }^{23}$, M. Pilar Ochoa ${ }^{24}$, Rafael López Castro ${ }^{25}$, Berta Obispo $^{26}$, Cristina Pangua ${ }^{26}$, María Sereno ${ }^{27}$, Lourdes Fernández Franco ${ }^{28}$, Xabier Mielgo ${ }^{29}$, Julia Calzas ${ }^{30}$, Ana Blasco ${ }^{31}$, Francisco Aparisi ${ }^{32}$, Luis Chara ${ }^{33}$, Juan Francisco Grau, Marta Soares ${ }^{5}$, Ana Gómez ${ }^{6}$, Victor Zenzola ${ }^{7}$, Marcial García-Morillo ${ }^{14}$, Diego Cacho $^{13}$, Asunción Díaz-Serrano ${ }^{1,2}$, Carlos Aguado ${ }^{10}$, Santiago Ponce-Aix ${ }^{1,2}$, Jose Luis González-Larriba ${ }^{10}$, Andrés J. Muñoz ${ }^{12}$, David Lora $\overbrace{}^{34}$, Luis Paz-Ares ${ }^{1,2,3}$ and Aránzazu Manzano ${ }^{10,35}$

${ }^{1}$ Medical Oncology Dept, Hospital Universitario 12 de Octubre and i+12 Research Institute, Madrid, Spain. ${ }^{2}$ Lung Cancer Group, Clinical Research Program, Spanish National Cancer Research Center (CNIO), Madrid, Spain. ${ }^{3}$ Centro de Investigación Biomédica en Red de Cáncer (CIBERONC), Spain. ${ }^{4}$ Medical Oncology Dept, Hospital Universitario Vall d'Hebrón, Barcelona, Spain. ${ }^{5}$ Medical Oncology Dept, Portuguese Institute of Oncology of Porto, Porto, Portugal. ${ }^{6}$ Medical Oncology Dept, Hospital Universitario Ramón y Cajal, Madrid, Spain. ${ }^{7}$ Medical Oncology Dept, Hospital Universitario Fundación Jiménez Díaz, Madrid, Spain. ${ }^{8}$ Medical Oncology Dept, Instituto Catalán de Oncología, L'Hospitalet, Barcelona, Spain. ${ }^{9}$ Clinical Research in Solid Tumors (CReST) Group, OncoBell Program, IDIBELL, L'Hospitalet, Barcelona, Spain. ${ }^{10}$ Medical Oncology Dept, Hospital Universitario Clínico San Carlos, Madrid, Spain.

${ }^{11}$ Medical Oncology Dept, Complejo Hospitalario de Navarra, Pamplona, Spain. ${ }^{12}$ Medical Oncology Dept, Hospital General Universitario Gregorio Marañón, Madrid, Spain. ${ }^{13}$ Medical Oncology Dept, Hospital Universitario Marqués de Valdecilla, Santander, Spain. ${ }^{14}$ Medical Oncology Dept, Hospital Clinic Barcelona, Barcelona, Spain. ${ }^{15}$ Medical Oncology Dept, Hospital Universitario Puerta de Hierro, Madrid, Spain. ${ }^{16}$ Medical Oncology Dept, Hospital Universitario La Paz, Madrid, Spain. ${ }^{17}$ Medical Oncology Dept, Hospital Universitario Virgen del Rocío-Clínica Oncoavanze, Sevilla, Spain. ${ }^{18}$ Medical Oncology Dept, Complejo Hospitalario Universitario de Albacete, Albacete, Spain. ${ }^{19}$ Medical Oncology Dept, Hospital Universitario La Princesa, Madrid, Spain. ${ }^{20}$ Medical Oncology Dept, Complejo Hospitalario Universitario de Cáceres, Cáceres, Spain. ${ }^{21}$ Medical Oncology Dept, Hospital Universitari i Politècnic La Fe, Valencia, Spain. ${ }^{22}$ Hematology and Medical Oncology Dept, Hospital General Universitario Morales Messeguer, Murcia, Spain. ${ }^{23}$ Medical Oncology Dept, Hospital Universitario Virgen de las Nieves, Granada, Spain. ${ }^{24}$ Medical Oncology Dept, Hospital Central de la Defensa 'Gómez Ulla', Madrid, Spain. ${ }^{25}$ Medical Oncology Dept, Hospital Clínico Universitario, Valladolid, Spain. ${ }^{26}$ Medical Oncology Dept, Hospital Universitario Infanta Leonor, Vallecas, Spain. ${ }^{27}$ Medical Oncology Dept, Hospital Universitario Infanta Sofía, Madrid, Spain. ${ }^{28}$ Medical Oncology Dept, Hospital Virgen de la Salud, Toledo, Spain. ${ }^{29}$ Medical Oncology Dept, Hospital Universitario Fundación Alcorcón, Madrid, Spain. ${ }^{30}$ Medical Oncology Dept, Hospital Universitario de Fuenlabrada, Madrid, Spain. ${ }^{31}$ Medical Oncology Dept, Hospital General Universitario de Valencia, Valencia, Spain. ${ }^{32}$ Medical Oncology Dept, Hospital Virgen de los Lirios de Alcoy, Alicante, Spain. ${ }^{33}$ Medical Oncology Dept, Hospital Universitario de Guadalajara, Guadalajara, Spain. ${ }^{34}$ Epidemiology and Public Health Dept, CIBERESP (i+12), Hospital Universitario 12 de Octubre, Madrid, Spain.

${ }^{35}$ These authors contributed equally to this work.

Correspondence: Jon Zugazagoitia, Servicio de Oncología Médica, Hospital Universitario 12 de Octubre, Av. de Córdoba Km 5.4, 28041 Madrid, Spain. E-mail: jonzuga@gmail.com 
Received: Aug 082017 | Accepted after revision: Feb 242018

Author contributions: J. Zugazagoitia, A. Manzano, L. Paz-Ares, S. Ponce-Aix and A.J. Muñoz conceived and designed the study. All authors were involved in data collection. D. Lora, together with J. Zugazagoitia and A. Manzano, did the statistical analysis. J. Zugazagoitia, A. Manzano, D. Lora and L. Paz-Ares contributed to data interpretation. J. Zugazagoitia and A. Manzano, together with L. Paz-Ares, wrote the original draft of the manuscript. All authors participated in manuscript writing and editing, and approved the final version of the article.

Conflict of interest: M. Biosca has received personal fees from Leo-Pharma and Sanofi, outside the submitted work. L. Paz-Ares has received honoraria from Roche, Lilly, Bristol Meyers Squibb, Merck \& Sharp D., Boehringer Ing., Pfizer, AstraZeneca, Amgem and Novartis, outside the submitted work. A. Manzano has received personal fees from Pharmamar, Roche and AstraZeneca, outside the submitted work.

Support statement: The Thrombosis Working Group of the Spanish Society of Medical Oncology supported the study and contributed to the distribution of the protocol to participating centres. M. Biosca, C. Font, I. García Escobar, C. Salvador-Coloma, M. Sánchez Cánovas, B. Obispo, J. Calzas, A.J. Muñoz and A. Manzano are members of the Thrombosis Working Group of the Spanish Society of Medical Oncology. J. Zugazagoitia was funded by Instituto de Salud Carlos III (Rio Hortega, CM15/00196). L. Paz-Ares was funded by ISCIII: PI1401964, PIE15/00076, RTICC (R12/ 0036/0028) and CIBERONC (C16/12/00442), co-funded by FEDER from Regional Development European Funds (European Union)

\section{References}

1 Blom JW, Doggen CJM, Osanto S, et al. Malignancies, prothrombotic mutations, and the risk of venous thrombosis. JAMA 2005; 293: 715-722.

2 Tagalakis V, Levi D, Agulnik JS, et al. High risk of deep vein thrombosis in patients with non-small cell lung cancer: a cohort study of 493 patients. J Thorac Oncol 2007; 2: 729-734.

3 Hicks LK, Cheung MC, Ding K, et al. Venous thromboembolism and nonsmall cell lung cancer: a pooled analysis of National Cancer Institute of Canada Clinical Trials Group trials. Cancer 2009; 115: 5516-5525.

4 Blom JW, Osanto S, Rosendaal FR. The risk of a venous thrombotic event in lung cancer patients: higher risk for adenocarcinoma than squamous cell carcinoma. J Thromb Haemost 2004; 2: 1760-1765.

5 Numico G, Garrone O, Dongiovanni V, et al. Prospective evaluation of major vascular events in patients with nonsmall cell lung carcinoma treated with cisplatin and gemcitabine. Cancer 2005; 103: 994-999.

6 Connolly GC, Menapace L, Safadjou S, et al. Prevalence and clinical significance of incidental and clinically suspected venous thromboembolism in lung cancer patients. Clin Lung Cancer 2013; 14: 713-718.

7 Alexander M, Kirsa S, Wolfe R, et al. Thromboembolism in lung cancer - an area of urgent unmet need. Lung Cancer 2014; 84: 275-280.

8 Jordan EJ, Kim HR, Arcila ME, et al. Prospective comprehensive molecular characterization of lung adenocarcinomas for efficient patient matching to approved and emerging therapies. Cancer Discov 2017; 7: 596-609.

9 Verso M, Chiari R, Mosca S, et al. Incidence of CT scan-detected pulmonary embolism in patients with oncogene-addicted, advanced lung adenocarcinoma. Thromb Res 2015; 136: 924-927.

10 Zer A, Moskovitz M, Hwang DM, et al. ALK-rearranged non-small-cell lung cancer is associated with a high rate of venous thromboembolism. Clin Lung Cancer 2017; 18: 156-161.

11 Khorana AA, Kuderer NM, Culakova E, et al. Development and validation of a predictive model for chemotherapy-associated thrombosis. Blood 2008; 111: 4902-4907.

12 Fine JP, Gray RJ. A proportional hazards model for the subdistribution of a competing risk. J Am Stat Assoc 1999; 94: 496-509.

13 Khorana AA, Francis CW, Culakova E, et al. Thromboembolism is a leading cause of death in cancer patients receiving outpatient chemotherapy. J Thromb Haemost 2007; 5: 632-634.

14 Gainor JF, Tan DSW, De Pas T, et al. Progression-free and overall survival in ALK-positive NSCLC patients treated with sequential crizotinib and ceritinib. Clin Cancer Res 2015; 21: 2745-2752.

15 Peters S, Camidge DR, Shaw AT, et al. Alectinib versus crizotinib in untreated ALK-positive non-small-cell lung cancer. N Engl J Med 2017; 377: 829-838. 